
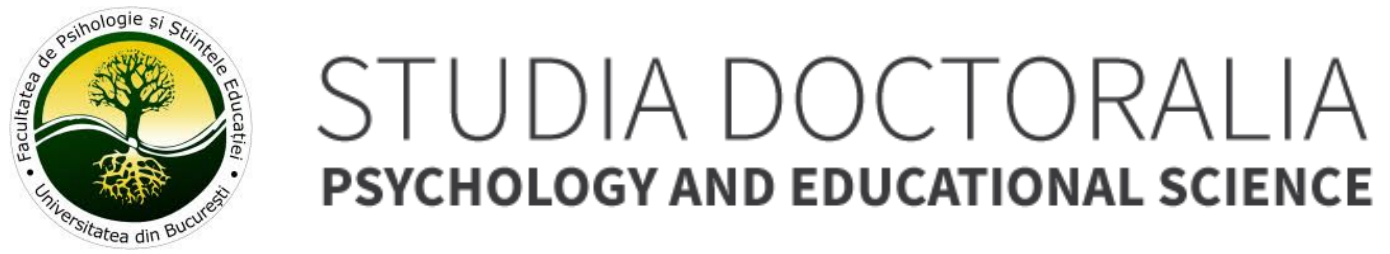

PSYCHOLOGY AND EDUCATIONAL SCIENCE

\title{
The Academic Motivation Scale (AMS): Factorial Structure, Validity and Reliability of The Romanian Version
}

\author{
Amalia Miulescu \\ University of Bucharest
}

\section{ARTICLE INFO}

Article history:

Received 10-September-2018

Accepted 15-December-2018

Available online 01-May-2019

This article should be cited as: Miulecu, A. (2019). The Academic Motivation Scale (AMS): Factorial Structure, Validity and Reliability of The Romanian Version. Studia Doctoralia. Psychology and Educational Science, 10, 29-40.

This is an open access article under the CC BY license (http://creativecommons.org/licenses/by/4.0/).

Corresponding author at: University of Bucharest, Department of Psychology, 90 Panduri Av, Bucharest, RO.

E-mail address: amalia.miulescu@gmail.com

\section{ABSTRACT}

This study presents the Romanian adaptation of The Academic Motivation Scale (AMS). The AMS scale was based on Self-Determination Theory (Ryan \& Deci, 1985) which identifies, along a continuum, from not self determined behavior to self - determined, 3 different types of motivation: amotivation, extrinsic motivation and intrinsic motivation, grouped in seven dimensions. Two studies were conducted to examine the factorial structure and validity (i.e., construct, concurrent and predictive validity) of the scale along with its reliability properties. For study 1 , the results obtained from a sample that included 228 students from the University of Bucharest, confirmed the 7 factors structure of the scale and verified adequate psychometric properties of this version (Cronbach alpha ranged between .69 to .87 with a mean of .81). Study 2, evaluated the temporal stability using a sample of 107 participants ( $r$ testretest, ranged from .52 to .69). The findings of the present study showed that AMS is a valid measure and can be used in the Romanian cultural area.

Keywords: academic motivation, self-determination theory, amotivation, extrinsic motivation, intrinsic motivation

\section{INTRODUCTION}

One of the topics most studied in educational psychology is academic motivation as it plays a major role in human behavior with important implications in understanding the forces that drive people towards taking actions and accomplishing things. This study is an attempt to provide a consistent tool in assessing motivation in academic milieu, 
based on Deci and Ryan's (1985) Self Determination Theory (SDT). The theory is centered around "why" individuals rise to action, identifying a continuum from lack of determination (unmotivated) to self-determination (intrinsic motivated), distinguishing between different types of motivation based on the different reasons or goals that give rise to an action
(Ryan \& Deci, 2000). SDT makes a clear distinction between intrinsic motivation, which refers to acting because something is pleasant and brings satisfaction just by experience itself, and extrinsic motivation which is oriented for a distinct reward, other than the activity.

Table 1 - Self-determination continuum based on Deci and Ryan (1985) and Vallerand et al.(1992)

\begin{tabular}{|c|c|c|c|c|c|c|c|c|}
\hline Behavior & \multicolumn{6}{|c|}{ Not self-determined } & \multicolumn{2}{|c|}{ Self-determined } \\
\hline Motivation type & \multicolumn{2}{|l|}{ Amotivation } & \multicolumn{3}{|c|}{ Extrinsic motivation } & \multicolumn{3}{|c|}{ Intrinsic motivation } \\
\hline Regulation styles & No regulation & $\begin{array}{l}\text { External } \\
\text { regulation }\end{array}$ & $\begin{array}{l}\text { Introjected } \\
\text { regulation }\end{array}$ & $\begin{array}{l}\text { Identified } \\
\text { regulation }\end{array}$ & $\begin{array}{l}\text { Integrated } \\
\text { regulation }\end{array}$ & & Intrinsic regi & \\
\hline AMS & Am & EM_er & EM_int & EM_id & & IM_k & IM_achiv & IM_se \\
\hline
\end{tabular}

Note: AMS, Academic Motivation Scale; Am, Amotivation; EM_er, Extrinsic Motivation external regulation; EM_int, Extrinsic Motivation introjected regulation; EM_id, Extrinsic Motivation identified regulation; IM_k, Intrinsic Motivation orientation towards knowledge; IM_achiv, Intrinsic Motivation orientation towards achievement; IM_se, Intrinsic Motivation orientation towards stimulating experiences:

\section{Self-determination theory}

According to Ryan and Deci (2000), the factors that enhance versus undermine intrinsic motivation, self-regulation, and well-being have been identified as three innate psychological needs: competence, autonomy and relatedness. Autonomy refers to the people's need to feel that they independently choose their actions, relatedness is about having connections with others and competence appears when they feel they have the abilities to act successfully. People would be at their best when these needs are satisfied, as motivation, performance and development are enabled.

When a behavior is self-determined, the regulatory process is choice, but when it is controlled, the regulatory process is compliance (or in some cases defiance) (Deci, Vallerand, Pelletier, \& Ryan, 1991).

Amotivation (AM) is positioned at the not self-determined extreme of the motivation continuum and it is characterized by very low level of autonomy and absence of purpose. Individuals who are amotivated lack an intention to act (Ryan \& Deci, 2000): they are neither intrinsically nor extrinsically motivated and they also believe their actions are the result of something that is beyond their control (Vallerand et al., 1992). Extrinsic motivation - external regulation (EM_er) follows amotivation on the continuum and is defined by actions that are reinforced by others in order to obtain rewards or to avoid punishment. Introjected regulation (EM_int) is the first form of internalized motivation but not truly self-determined as it refers to improving one's selfesteem and feeling of worth. In identified regulation (EM_id) people internalize the purpose of the activity and the value of their learning activity. The most internalized form of extrinsic motivation is integrated regulation, in which people integrate their behavior but still they act for an external outcome distinct from their behavior. This form of extrinsic motivation appears late in the adult life and for that reason it wasn't included in AMS scale. To the other extreme of the continuum is placed intrinsic motivation, the autonomous form of motivation, where the satisfaction of experiencing different activities by own choice prevails: activities themselves become a goal. Several years later, Vallerand et al. (1989) identified 3 dimensions of the intrinsic motivation: to know (IM_k), towards achievement (IM_achiv) and towards stimulating experiences (IM_se). Intrinsic motivation - orientation towards knowledge (IM_k) is related to the pleasure of learning, exploration and curiosity. Orientation towards achievement (IM_achiv) can be defined as the satisfaction of engaging in an activity and surpassing one's limits. And finally, orientation towards stimulating experiences (IM_se) involves activities that people perceive to lead to stimulating experiences such as: aesthetics, intellectual or inducing excitement.

The AMS scale was developed in order to identify the types of motivation from this continuum and to predict different behaviors that could lead to positive outcomes (e.g., academic performance) but also to predict negative ones (e.g., drop out of school).

A 7-factor solution has been replicated using samples from many different countries, including Canada (Vallerand et al. 1993; Vallerand et al., 1992), the United States (Fairchild, Horst, Finney \& Barron, 2005), Greece (Barkoukis, Tsorbatzoudis, Grouios, \& Sideridis, 2008), Norway (Støen Utvær \& Haugan, 2016), Argentina (Stover, de la Iglesia, Rial, \& Fernández Liporace, 2012), Iran (Taghipour et al., 2012) and Turkey (Can, 2015). The Italian version of the scale was developed on a 5 factors structure (Alivernini \& Lucidi, 2008). Therefore, the validation of a theory-based instrument such as AMS in states with different cultures would offer further support on the generalization of the SDT theory (Barkoukis, Tsorbatzoudis, Grouios, \& Sideridis, 2008). 


\section{Related concepts}

Academic performance is strongly influenced by individual differences in terms of personality traits, as well as by motivation and goal orientation. The relation between Big five-model and motivation has provided a framework that showed a strong relation between conscientiousness and the central aspect of motivation-self-regulation (Barrick, Mount, \& Strauss, 1993). Therefore, a significant correlation between consciousness (C) and IM is expected to arise.

The AGQ scale was conceptualized as a cognitive-dynamic scale that aims on two competence dimensions: approachavoidance achievement motivation. Competence can be defined in terms of the standard used to evaluate it, that is, relative to an absolute or intrapersonal standard (mastery) or relative to a normative standard (performance) (Elliot \& Murayama, 2008). IM and EM subscales are expected to show a significant medium to high correlations with MasteryApproach and Performance-approach scales while Am subscale is expected to show negative correlations with Mastery-approach and Mastery-avoidance scales, as these scales involve an internal locus of control and sense of competence, opposite to Am.

The core self-evaluation model includes four traits: neuroticism, self-esteem, locus of control, and generalized

\section{METHODOLOGY}

\section{Power Analysis}

In order to determine the optimal sample needed to perform this study, power analysis was performed using GPower. Thus, to detect medium effect sizes (.30 or greater) with a power of .80 and 351 degrees of freedom, the optimal sample required for the evaluation of the AMS scale comprising 28 items, grouped into 7 factors, is 793 participants. In this study, this standard was not reached, the sample used consisted of 228 participants for study 1 and 107 participants for study 2 .

\section{Participants}

Study 1. The sample consisted of 228 college students from the University of Bucharest, Faculty of Psychology and Educational Sciences, 32 men (14\%) and 196 women (86\%), aged 17-48 (mean 20.20, SD 4.84). The students were enrolled in the first year of study, data collection was made in November 2017. Only one application was made, which made it necessary to carry out a new study (Study 2) to measure stability over time through test-retest reliability.

Study 2. The sample used to measure the test-retest reliability consisted of 107 participants from the University of Bucharest, Faculty of Psychology and Educational Sciences. The participants were students enrolled in bachelor's and master's programs, 14 men (13\%) and 93 women (87\%) aged 17 to 47 (average 22.11, SD 5.15). Data collection was conducted in December 2018 - the first. self-efficacy. In their research, Erez and Judge (2001), revealed that core self-evaluations was related to motivation and performance. Also, they showed that personality variables are related to motivation to perform through selfregulatory mechanisms as individuals with high levels of core self-evaluations would be more motivated to perform and exhibit higher levels of task performance. SDT describes intrinsically motivated individuals as having an internal locus of control, driven to accomplish or to seek intellectual stimulation and enjoy learning new things, while extrinsically motivated individuals pursue education to achieve contingent goals, rather than for an intrinsic enjoyment of achievement. As a consequence, Am, EM_er and EM_int are expected to show a negative correlation with core self-evaluation, while Em_id and IM subscales are expected to record positive ones.

(needs).

\section{Research objectives}

The general objective of the present study was Romanian adaptation of AMS scale. Specific objectives were adequate translation of the items, reliability analysis, criterion validity and construct validity assessed by confirmatory factor analysis on a Romanian sample.

\section{Procedure}

For both studies, the informed consent of the participants was obtained, written instructions were given to them, regarding the completion of the scale and they were assured about the confidentiality of their responses. For study 1 , subjects completed the Romanian versions of AMS, AGQ, CSES and Consciousness online through Google platform. Completing the questionnaires took about 20 minutes.

For Study 2, the participants completed twice the Romanian version of AMS via Google platform, over a period of 6 to 10 weeks. The completion of the questionnaire took approximately 10 minutes and 5 prizes were offered to the participants, consisting of psychology books. The prizes were randomly assigned by random.org.

\section{Measurement}

The Romanian version of AMS was used after the items were translated into Romanian and then back-translated into English to ensure an adequate adaptation. The scale consists of 28 possible answers to the question: "Why do you go to college?", grouped in seven factors with four items each: amotivation (e.g.: "I once had good reasons for going to college; however, now I wonder whether I should continue"), external regulation (e.g.: "In order to obtain a more prestigious job later on"), introjection (e.g.: "Because I want to show myself that I can succeed in my studies"), identification (e.g.: "Because this will help me make a better 
choice regarding my career orientation"), intrinsic motivation to know (e.g.: "For the pleasure I experience when I discover new things never seen before"), intrinsic motivation towards accomplishment (e.g.: "For the satisfaction I feel when I am in the process of accomplishing difficult academic activities") and intrinsic motivation to experience stimulation (e.g.: "For the pleasure that I experience when I read interesting authors"). All items are scored on a 7-point rating scale ranging from 1 ("Not at all") to 7 ("Exactly") with a midpoint at 4 ("Moderately") (Vallerand et al., 1992).

Core Self Evaluation Scale (CSES; Judge, Erez, Bono, \& Thoresen, 2003) is a unidimensional scale which consists of 12 items (e.g., "I am confident I get the success I deserve in life", Cronbach's a =.87), scored on a 5-point rating scale ranging from 1 ("totally disagree") to 5 ("totally agree").

Achievement Goal Questionnaire (AGQ; Elliot \& Murayama, 2008) has 12 items: mastery-approach goal items (3 items,

\section{RESULTS}

\section{Study 1}

\section{Construct validity}

Analyses were conducted via SPSS 25 (IBM Corp. Released 2017) and MPlus 7.0 (Muthén, \& Muthén, 19982012). Confirmatory factor analysis was used in order to test the factorial validity of the scale. For analyzing the normality e.g., "My aim is to completely master the material presented in this class", Cronbach's a =.66), mastery-avoidance goal items ( 3 items, e.g., "My aim is to avoid learning less than I possibly could", Cronbach's a =.69), performance-approach goal items ( 3 items, e.g., "My aim is to perform well relative to other students.", Cronbach's $a=.88$ ) and performanceavoidance goal items ( 3 items, e.g., "My aim is to avoid doing worse than other students.", Cronbach's $a=.90$ ). Responses were given on a 5-point Likert scale ranging from 1 ("totally disagree") to 5 ("totally agree").

Consciousness scale from the IPIP -50 Questionnaire (Goldberg et al., 2006; lliescu, Popa \& Dimache, 2015) consisted of 10 items (e.g., "Pay attention to details", Cronbach's $a=.81$ ) scored on a 5-point rating scale ranging from 1 ("totally disagree") to 5 ("totally agree").

The predictive validity was evaluated by results obtained at the end of the first semester.

of the distribution, standardized values of skewness and kurtosis were evaluated for each item, in addition to their means and standard deviations. As

presented in Table 1, most items had values over \pm 1.96 , therefore, the null hypothesis of normal distribution was rejected. As a consequence, the method of choice for the factorial study was robust Maximum Likelihood (MLM) since, according to Byrne (2013), citing Muthén, \& Muthén (20072010), this method is appropriate for various distributions and sample sizes. 
Table 1. Item analysis and descriptive statistics

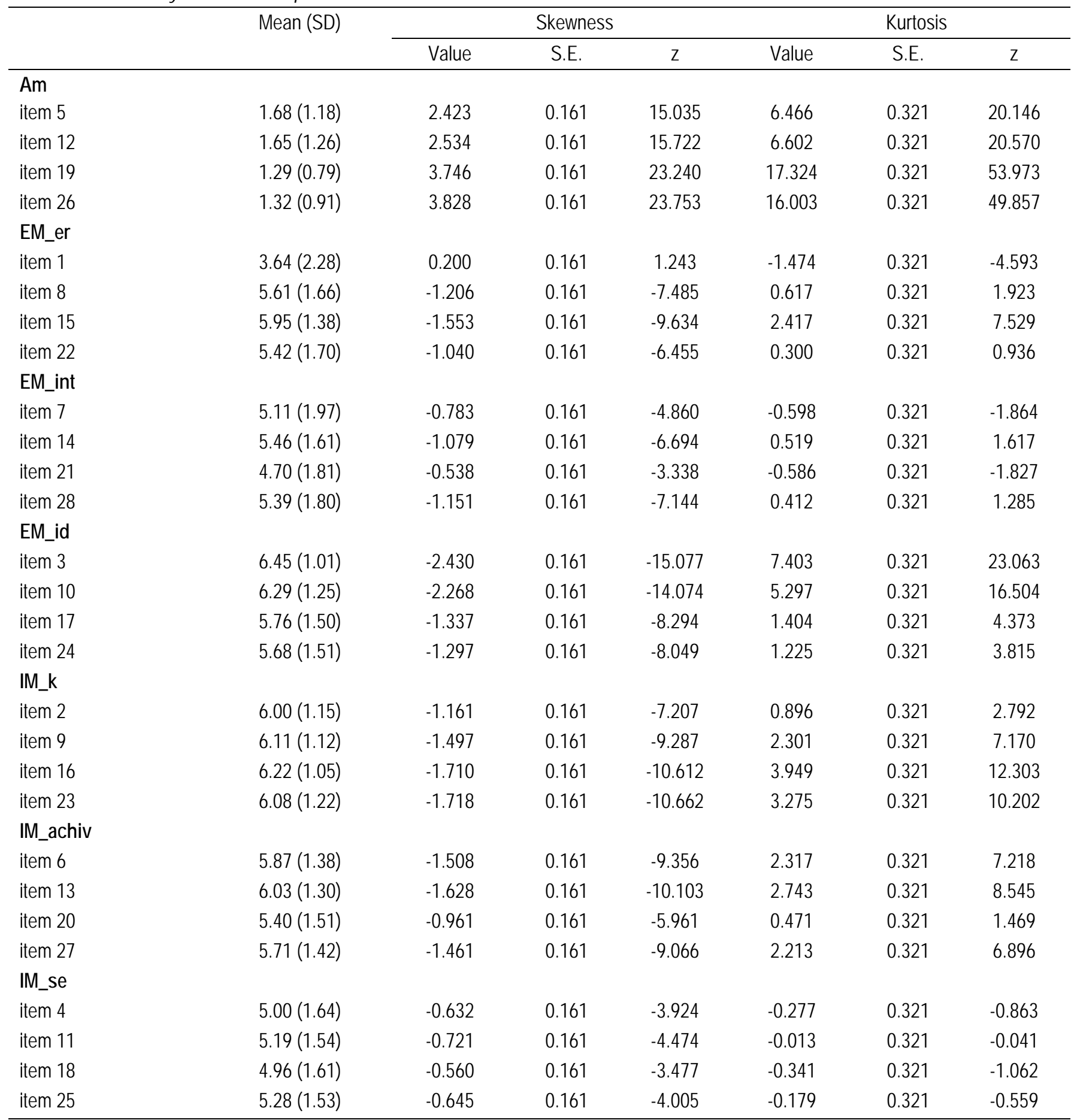

Note: Am, Amotivation; EM_er, Extrinsic Motivation external regulation; EM_int, Extrinsic Motivation introjected regulation; EM_id, Extrinsic Motivation identified regulation; IM_k, Intrinsic Motivation orientation towards knowledge; IM_achiv, Intrinsic Motivation orientation towards achievement; IM_se, Intrinsic Motivation orientation towards stimulating experiences;

For testing the goodness-of-fit, the comparative fit index (CFI) was used as the main index, because of its appropriate statistical properties, such as its standardized 0-1 range, small sample variability, and stability with various sample sizes (Bentler 1990). According to Hu and Bentler (1999), CFI higher than .95 represents adequate model fit, however, values above .90 have also been considered acceptable (Bentler, 1990).

There were analyzed different models, starting with 1-factor model, 3-factors model, 5-factors model, and finally 7factors model. As expected, the 7-factor model showed better fit values. The initial 7-factors model didn't show an acceptable fit $(\mathrm{CFI}=.88$; RMSEA $=.07$; SRMR $=.08)$. 


\section{Model revision}

After analyzing the Modification Indices section, in the model were included 7 correlated pairs of measured variables residuals, item11 with item18 (M.I. = 29.21), item13 with item6 (M.I. = 20.46), item20 with item18 (M.I. = 16.31), item28 with 17 (M.I. = 14.78), item28 with item2 (M.I. =
16.31), item6 with item2 (M.I. = 13.31) and item23 with item13 (M.I. = 12.29) were included in the model. With these changes, the model showed an acceptable fit (CFI $=.92$; RMSEA $=.05$; SRMR $=.07$ ). The final results are presented in Table 2.

Table 2. CFA results

\begin{tabular}{|c|c|c|c|c|c|c|c|}
\hline AMS & ${ }^{*} \lambda^{2}$ & df & $\begin{array}{l}\Lambda^{2} / \mathrm{df} \\
\text { ratio }\end{array}$ & $\mathrm{CFI}$ & TLI & SRMR & $\begin{array}{l}\text { RMSEA } \\
(90 \% \mathrm{Cl})\end{array}$ \\
\hline 1 factor & $1741.87^{\star}$ & 351 & 4.963 & .47 & .43 & .16 & $.13(.13-.14)$ \\
\hline 3 factors & $1072.90 *$ & 347 & 3.092 & .73 & .70 & .11 & $.10(.09-.10)$ \\
\hline 5 factors & $856.64^{\star}$ & 340 & 2.250 & .80 & .78 & .10 & $.08(.08-.09)$ \\
\hline 7 factors & $530.95^{\star}$ & 322 & 1.650 & .92 & .91 & .07 & $.05(.05-.06)$ \\
\hline
\end{tabular}

Note: ${ }^{*}=p<.001 ;{ }^{*}$ X = Satorra-Bentler chi square; $d f$ = degrees of freedom; CFI = Comparative Fit Index; TLI = Tucker-Lewis Index; SRMR = Standardized Root Mean Square Residual; RMSEA = Root Mean Squared Error of Approximation 


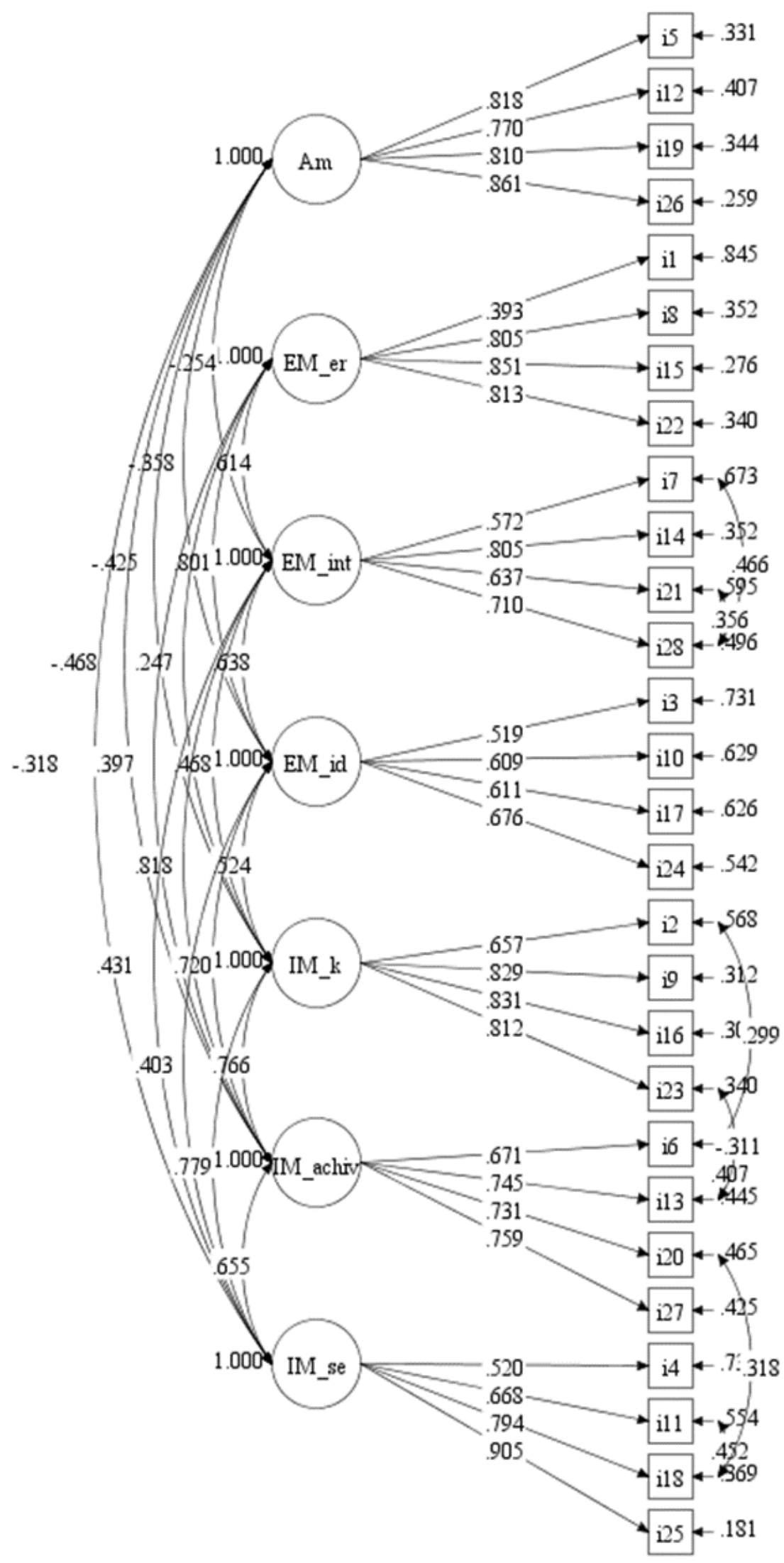

Figure 2. Standardized estimated parameters. $A m=$ Amotivation; $E M$ er $=$ Extrinsic Motivation external regulation; $E M$ int $=$ Extrinsic Motivation introjected regulation; EM_id = Extrinsic Motivation identified regulation; IM_k = Intrinsic Motivation orientation towards knowledge; IM_achiv = Intrinsic Motivation orientation towards achievement; IM_se = Intrinsic Motivation orientation towards stimulating experiences 
In most cases, the estimated values for the correlations between factors, were significant except the correlation between Am with EM_er factors, which was not significant (Figure 2).

Correlations analysis were conducted for the AMS subscales. The results were in the expected direction, more specifically, the IM sub-scales demonstrated medium to high correlations with each other ( $r$ range: .27 to .66), low to high correlations with the EM sub-scales ( $r$ range: 16 to .53 ) and low negative correlations with amotivation ( $r$ range: -.24 to -.38). Similarly, the EM sub-scales showed medium to high correlations with each other ( $r$ range: .44 to .57), and low and negative correlations with amotivation ( $r$ range: - .14 to .28) Only the relation between the IM to achievement and two of the EM sub-scales: introjection and identification was higher than the expected values ( $r$ range: .51 to .53 ) (Table 3).

Table 3. Descriptive Statistics and correlations between the AMS subscales

\begin{tabular}{|c|c|c|c|c|c|c|c|c|c|}
\hline & Means & SD & 1 & 2 & 3 & 4 & 5 & 6 & 7 \\
\hline 1. Am & 5.94 & 3.58 & $(.87)$ & & & & & & \\
\hline 2. EM_er & 20.61 & 5.46 & $.15^{\star}$ & $(.76)$ & & & & & \\
\hline 3. EM_int & 20.66 & 5.90 & $-.14^{\star}$ & $.44^{\star *}$ & (.84) & & & & \\
\hline 4. EM_id & 24.18 & 3.84 & $-.28^{\star}$ & $.57^{\star \star}$ & $.47^{\star \star}$ & (.69) & & & \\
\hline 5. IM_k & 24.41 & 3.83 & $-.37^{\star}$ & $.16^{*}$ & $.31^{\star \star}$ & $.43^{\star \star}$ & (.86) & & \\
\hline 6. IM_achiv & 23.00 & 4.58 & $-.38 *$ & $.22^{\star}$ & $.53^{\star \star}$ & $.51^{\star \star}$ & $.66^{\star \star}$ & (.83) & \\
\hline 7. IM_se & 20.43 & 5.12 & $-.24^{*}$ & .003 & .30 ** & .30 ** & $.66^{\star *}$ & $.56^{\star \star}$ & $(.82)$ \\
\hline
\end{tabular}

Note. ${ }^{*} p<.05 \quad{ }^{* \star} p<.01 \quad$ Am, Amotivation; EM_Er, Extrinsic Motivation external regulation; EM_int, Extrinsic Motivation introjected regulation; EM_id, Extrinsic Motivation identified regulation; IM_k, Intrinsic Motivation orientation towards knowledge; IM_achiv, Intrinsic Motivation orientation towards achievement; IM_se, Intrinsic Motivation orientation towards stimulating experiences; Numbers in parentheses represent Cronbach alpha values.

These findings come to support the Self Determination Theory in the sense that the more distal the constructs are on the continuum of self-determination, the lower are the correlations between them, and thus, provides strong support regarding the construct validity of the scale (Vallerand et al. 1993).

\section{Criterion validity}

Criterion validity was evaluated through concurrent and predictive validity.
For assessing concurrent validity, correlation analysis was conducted in relation with AGQ and CSES, as concurrent validity according to Kerlinger and Lee (1999) is evaluated by correlating two sets of scores (the new test - AMS, and existing tests, AGQ and CSES). Predictive validity was evaluated with academic performance at the end of the first semester, and divergent/discriminant validity was assessed in relation with Consciousness. These correlations were, with a few exceptions, in the expected direction (Table 4).

Table 4. Correlations between the variables included in the study

\begin{tabular}{|c|c|c|c|c|c|c|c|}
\hline & AGQ_m_app & AGQ_m_av & AGQ_p_app & AGQ_p_av & Core_Self & $C$ & $\mathrm{AP}$ \\
\hline Am & $-.22^{\star \star}$ & $-.18^{\star \star}$ & -.05 & -.02 & $-.19 \star \star$ & -.08 & -.08 \\
\hline EM_er & $.13^{\star}$ & .10 & $.29^{\star \star}$ & $.26^{\star \star}$ & -.02 & .05 & $.15^{\star}$ \\
\hline EM_int & $.34^{\star *}$ & $.14^{*}$ & $.41^{\star \star}$ & $.33^{\star \star}$ & $-.17^{\star}$ & -.04 & .12 \\
\hline EM_id & $.28^{\star *}$ & $.20^{\star \star}$ & $.22^{\star \star}$ & $.16^{\star}$ & .12 & .05 & $.20^{\star \star}$ \\
\hline IM_k & $.48^{\star \star}$ & $.29^{\star \star}$ & .10 & .07 & $.19^{\star \star}$ & $.18^{\star *}$ & $.15^{\star}$ \\
\hline IM_achiv & $.47^{\star \star}$ & $.32^{\star \star}$ & $.31^{\star \star}$ & $.25^{\star \star}$ & $.14^{\star}$ & $.16^{\star}$ & $.20^{\star \star}$ \\
\hline IM_se & $.48^{\star \star}$ & $.28^{\star \star}$ & .08 & .07 & .11 & $.15^{\star}$ & .04 \\
\hline
\end{tabular}

Note. ${ }^{*} p<.05 \quad{ }^{\star \star} p<.01$ Am, Amotivation; EM_er, Extrinsic Motivation external regulation; EM_int, Extrinsic Motivation introjected regulation; EM_id, Extrinsic Motivation identified regulation; IM_k, Intrinsic Motivation orientation towards knowledge; IM_achiv, Intrinsic Motivation orientation towards achievement; IM_se, Intrinsic Motivation orientation towards stimulating experiences; AGQ_m_app, Mastery-approach goal; AGQ_m_av, Mastery-avoidance goal; AGQ_p_app, Performance-approach goal; AGQ_p_av, Performanceavoidance goal; Core_Self, Core Self Evaluation; $C=$ Consciousness; $\bar{A} P$, Academic performance. 
Regarding concurrent validity, the AMS was correlated with the AGQ and CSES. The correlations with AGQ, as expected, the Mastery-approach goal subscale was showing negative low correlations with amotivation $(r=-.22)$, low to medium correlations with EM ( $r$ range: .13 to .34 ) and medium correlations with IM ( $\mathrm{r}$ range: .47 to .48). Mastery avoidance subscale was showing low correlation with amotivation ( $r=-.18)$, low correlations with EM - introjection and identification sub-scales $(r=.14$ and $r=.20$ respectively) and low to medium correlations with IM ( $\mathrm{r}$ range: .28 to .32). Performance-approach sub-scale showed low and medium correlations with extrinsic motivation $(r$ range: .22 to .41) and medium correlation with IM - towards achievement $(r=.31)$. Performance avoidance showed a low to medium correlations with EM ( $r$ range: .16 to .33) and surprisingly, a low positive correlation with IM - towards achievement $(r=.25)$. In relation with CSES, the results showed negative significant correlation with $\mathrm{Am}(r=-0.19)$ and EM_int $(r=-0.17)$, a negative but not significant correlation with EM_er $(r=-0.02)$, positive but not significant with EM_id $(r=0.12)$ and IM_se $(r=0.11)$ and positive significant correlations with IM_k $(r=0.19)$ and IM_achiv ( $r$ $=0.14$ ). These results are consistent with the theory as individuals with higher core self-evaluation are characterized by high self-esteem, generalized self-efficacy which denotes competence, low neuroticism which implies focus on the negative aspects of one self and locus of control which refers to beliefs about the causes of events, are more intrinsic motivated. Thus, AMS subscales in relation with CSES, showed negative correlations for Am, EM_er and EM_int and positive correlations for EM_id and IM subscales, showing that the more self-esteem, internal locus of control, self-efficacy and less neuroticism, individuals perceive about themselves, the more self-determined they are in their course of actions.

The predictive validity was tested in relation with academic performance at the end of first semester. The results indicated significant low correlations with EM - external regulation and identification sub-scales $(.15, .20)$ and also low correlations with IM - to know and towards achievement
$(.15, .20)$. In order to support the predictive validity, linear regression was conducted. The results showed that the simultaneous evaluation of all AMS subscales produced a significant $R^{2}=0.065$. Thus, the motivational orientations resulted in significant amounts of the variability of academic performance $(F(7,220)=2.18, p<.05)$.

For the discriminant/divergent validity, AMS was analyzed in relation with Consciousness scale. According to Campbell (1960), discriminant validity is "the requirement that a test not correlate too highly with measures from which it is supposed to differ". Thus, the results showed significant low correlations only with intrinsic motivation sub-scales of AMS $(.18, .16, .15)$. The fact that IM was correlating significantly with Consciousness, may be due to the fact that individuals who are more conscientious are also more disciplined and achievement oriented. These findings are consistent with the existing literature on personality traits and academic motivation (Komarraju, Karau, \& Schmeck, 2009).

\section{Reliability analysis}

Reliability has a direct impact on validity but it is also an important indicator of measurement error.

For study 1, given that there was only one measure of motivation, scale reliability was assessed by internal consistency which is based on inter-item correlations. AMS sub-scales have a good internal consistency with Cronbach's alpha ranging from .69 to .87 , similar to the values obtained by Valerand et al. (1992) on the Canadian sample (Table 5).

The Cronbach's alpha value for the ME-identification factor recorded a medium level (.69), but higher than the value obtained for the Canadian sample (.62). The other subscales of AMS showed high internal consistency.

\section{Study 2.}

The stability in time of the scale, measured through testretest reliability, was performed by correlation analysis. Thus, the results showed high correlations ranging between .52 and .69 between the two measurements (Table 5). 
Table 5. Internal consistency (Cronbach's alpha) for AMS - 7 factors; Test-retest correlations: Study 1 and Study 2

\begin{tabular}{|c|c|c|c|c|c|c|c|c|c|c|c|}
\hline \multirow[t]{4}{*}{ Factor } & \multirow[t]{4}{*}{ Items } & \multicolumn{7}{|c|}{ Romanian sample } & \multirow[t]{4}{*}{ Items } & \multirow{3}{*}{\multicolumn{2}{|c|}{$\begin{array}{l}\text { Canadian sample } \\
\qquad \mathrm{N}=745\end{array}$}} \\
\hline & & \multirow{2}{*}{\multicolumn{2}{|c|}{$\begin{array}{l}\text { Study } 1 \\
\mathrm{~N}=228\end{array}$}} & \multicolumn{5}{|c|}{ Study 2} & & & \\
\hline & & & & \multicolumn{4}{|c|}{$N=107$} & \multirow{2}{*}{$-\mathrm{T} 2^{\mathrm{T} 1}$} & & & \\
\hline & & Alpha & C.I. (95\%) & Alpha-T1 & C.I. (95\%) & Alpha-T2 & C.I. (95\%) & & & Alpha & C.I. $(95 \%)$ \\
\hline Amotivation & 4 & .87 & $.84-.90$ & .84 & $.78-.88$ & .88 & $.84-.92$ & .52 & 4 & .85 & - \\
\hline $\begin{array}{l}\text { Extinsic motivation - external } \\
\text { regulation }\end{array}$ & 4 & .76 & $.71-.81$ & .80 & $.73-.85$ & .79 & $.71-.85$ & .65 & 4 & .83 & - \\
\hline $\begin{array}{l}\text { Extinsic motivation - } \\
\text { introjected regulation }\end{array}$ & 4 & .84 & $.80-.87$ & .80 & $.72-.85$ & .84 & $.79-.89$ & .66 & 4 & .84 & - \\
\hline $\begin{array}{l}\text { Extinsic motivation - } \\
\text { identification }\end{array}$ & 4 & .69 & $.62-.75$ & .64 & $.51-.74$ & .76 & $.67-.82$ & .60 & 4 & .62 & - \\
\hline $\begin{array}{l}\text { Intrinsic motivation - } \\
\text { knowledge }\end{array}$ & 4 & .86 & $.83-.89$ & .85 & $.80-.89$ & .89 & $.85-.92$ & .66 & 4 & .84 & - \\
\hline $\begin{array}{l}\text { Intrinsic motivation - } \\
\text { achievement }\end{array}$ & 4 & .83 & $.79-.87$ & .84 & $.78-.88$ & .85 & $.80-.89$ & .57 & 4 & .85 & - \\
\hline $\begin{array}{l}\text { Intrinsic motivation - } \\
\text { stimulating experiences }\end{array}$ & 4 & .82 & $.78-.86$ & .82 & $.75-.87$ & .85 & $.80-.89$ & .69 & 4 & .86 & - \\
\hline
\end{tabular}




\section{CONCLUSIONS AND FURTHER RESEARCH}

The Academic Motivation Scale has been developed to answer the question of 'why' behind the motivated behavior, unlike the previous tools that focus on the antecedents and consequences of human behavior (Vallerand 1997).

The present study was started with the intention of validating and providing a measure for assessing motivation in the Romanian academic context that met required psychometric properties. The present findings remain coherent with those in the literature as the results support the factorial structure, reliability, and the predictive validity of the Romanian version of the AMS. The results confirmed the 7 factors structure of the scale and verified adequate psychometric properties of this version (Cronbach alpha ranged between .69 to .87 with a mean of .81). Beyond its factorial structure, AMS showed significant correlations with academic performance.

\section{REFERENCES}

Barkoukis, V., Tsorbatzoudis, H., Grouios, G., \& Sideridis, G. (2008). The assessment of intrinsic and extrinsic motivation and amotivation: Validity and reliability of the Greek version of the Academic Motivation Scale. Assessment in Education: Principles, Policy \& Practice, 15(1), 39-55. https://doi.org/10.1080/09695940701876128

Barrick, M. R., Mount, M. K., \& Strauss, J. P. (1993). Conscientiousness and performance of sales representatives: Test of the mediating effects of goal setting. Journal of Applied Psychology, 78, 715-722. http://dx.doi.org/10.1037/0021-9010.78.5.715

Bentler, P. M. (1990). Comparative fit indexes in structural models. Psychological bulletin, 107(2), 238. http://dx.doi.org/10.1037/0033-2909.107.2.238

Byrne, B. M. (2013). Structural equation modeling with Mplus: Basic concepts, applications, and programming. Routledge.

Hu, L., \& Bentler, P. M. (1999). Cutoff criteria for fit indexes in covariance structure analysis: Conventional criteria versus new alternatives. Structural Equation Modeling: $A$ Multidisciplinary Journal, 6(1), 1-55. https://doi.org/10.1080/10705519909540118

Campbell DT. (1960). Recommendations for APA test standards regarding construct, trait, or discriminant validity. American Psychologist, 15, 546-553. http://dx.doi.org/10.1037/h0048255

Can, G. (2015). Turkish version of the Academic Motivation Scale. Psychological Reports, 116(2), 388-408. doi: 10.2466/14.08.PR0.116k24w5

Deci, E. L., Vallerand, R. J., Pelletier, L. G., \& Ryan, R. M. (1991). Motivation and Education: The Self-Determination
From a theoretical perspective, given that it was stated that intrinsic forms of motivation are superior to the extrinsic forms (Ryan \& Deci, 1985), the use of a multidimensional measure of motivation should enable practitioners to identify the variables that undermine intrinsic motivation and also increase not-self-determined forms of motivation (such as amotivation and external regulation). Thus, it is important to pursue research on the determinants of the three types of $\mathrm{IM}$ and also of the consequences they lead to.

From a practical perspective, the use of AMS can lead to important findings regarding motivational changes during various educational programs.

However, further studies should be conducted on a larger sample size, as the current sample size was a moderate one $(n=228)$, and thus the results cannot be generalized.

In sum, the present findings provide support for the reliability and validity of the Romanian version of AMS.r

Perspective. Educational Psychologist, 26(3-4), 325-346. https://doi.org/10.1080/00461520.1991.9653137

Deci, E., \& Ryan, R. (1985). Intrinsic motivation and selfdetermination in human behaviour. New York: Plenum.

Elliot, A. J. \& Murayama, K. (2008). On the Measurement of Achievement Goals: Critique, Illustration, and Application. Journal of Educational Psychology, 100(3), 613-628. http://dx.doi.org/10.1037/0022-0663.100.3.613

Erez, A., \& Judge, T. A. (2001). Relationship of core selfevaluations to goal setting, motivation, and performance. Journal of Applied Psychology, 86(6), 1270-1279. https://doi.org/10.1037//0021-9010.86.6.1270

Fairchild, A.J., Horst, S.J., Finney, S.J., \& Barron, K.E. (2005). Evaluating existing and new validity evidence for the Academic Motivation Scale. Contemporary Educational Psychology, 30(3), 331-358.

http://dx.doi.org/10.1016/j.cedpsych.2004.11.001

Goldberg, L. R., Johnson, J. A., Eber, H. W., Hogan, R., Ashton, M. C., Cloninger, C. R., \& Gough, H. C. (2006). The International Personality Item Pool and the future of publicdomain personality measures. Journal of Research in Personality, 40, 84-96.

IBM Corp. Released 2017. IBM SPSS Statistics for Windows, Version 25.0. Armonk, NY: IBM Corp.

Iliescu, D., Popa, M., \& Dimache, R. (2015). The Romanian adaptation of the International Personality Item Pool: IPIPRo. Psihologia Resurselor Umane, 13(1), 83-112.

Judge, T. A., Erez, A., Bono, J. E., \& Thoresen, C. J. (2003). The Core Self-Evaluations Scale: Development of $A$ Measure. Personnel Psychology, 56(2), 303-331. https://doi.org/10.1111/j.1744-6570.2003.tb00152.x 
Kerlinger, F., Lee, H.B. (1999). Foundations of Behavioral Research. Wadsworth Publishing; 4 edition (August 9, 1999).

Komarraju, M., Karau, S. J., \& Schmeck, R. R. (2009). Role of the Big Five personality traits in predicting college students' academic motivation and achievement. Learning and Individual Differences, 19(1), 47-52. https://doi.org/10.1016/j.lindif.2008.07.001

Muthén, L.K. and Muthén, B.O. (1998-2012). Mplus User's Guide. Seventh Edition. Los Angeles, CA: Muthén \& Muthén Ryan, R. M., \& Deci, E. L. (2000). Intrinsic and Extrinsic Motivations: Classic Definitions and New Directions. Contemporary Educational Psychology, 25(1), 54-67. https://doi.org/10.1006/ceps.1999.1020

Ryan, R. M., \& Deci, E. L. (2000). Self-determination theory and the facilitation of intrinsic motivation, social development, and well-being. American Psychologist, 55(1), 68.

Støen Utvær, B. K., \& Haugan, G. (2016). The Academic Motivation Scale: Dimensionality, Reliability, and Construct Validity Among Vocational Students. Nordic Journal of Vocational Education and Training, 6(2), 17-45. https://doi.org/10.3384/njvet.2242-458X.166217

Stover, J., de la Iglesia, Rial, A., \& Fernández Liporace. (2012). Academic Motivation Scale: adaptation and psychometric analyses for high school and college students.
Psychology Research and Behavior Management, 71. https://doi.org/10.2147/PRBM.S33188

Taghipour, H. A., Gilaninia, S., Jalali, M., Azizipour, H., Razaghi, S. J. R., \& Mousavian, S. J. (2012). Standardizing of Academic Motivation Scale. Journal of Basic and Applied Scientific Research, 2(2)1186-1192.

Vallerand, R. J., Pelletier, L. G., Blais, M. R., Brière, N. M., Senecal, C., \& Vallières, É. F. (1993). On the assessment of intrinsic, extrinsic, and amotivation in education: Evidence on the concurrent and construct validity of the Academic Motivation Scale. Educational and Psychological Measurement, 53(1), 159-172.

Vallerand, R. J., Pelletier, L. G., Blais, M. R., Briere, N. M., Senecal, C., \& Vallieres, E. F. (1992). The Academic Motivation Scale: A Measure of Intrinsic, Extrinsic, and Amotivation in Education. Educational and Psychological Measurement, 52(4), 1003-1017. https://doi.org/10.1177/0013164492052004025

Vallerand, R. J., Blais, M. R., Brière, N. M., \& Pelletier, L. G. (1989). Construction et validation de l'échelle de motivation en éducation (EME) [Construction and validation of the Motivation toward Education Scale]. Canadian Journal of Behavioural Science / Revue canadienne des sciences du comportement, 21(3), 323-349.

http://dx.doi.org/10.1037/h0079855 